\title{
Products of Random Matrices for Disordered Systems
}

\author{
A. Crisanti \\ Dipartimento di Fisica, Università "La Sapienza", I-00185 Roma, Italy \\ G. Paladin \\ Dipartimento di Fisica, Università dell'Aquila, I-67010 Coppito, L'Aquila, Italy \\ M. Serva \\ Dipartimento di Matematica, Università dell'Aquila, I-67010 Coppito, L'Aquila, Italy \\ A. Vulpiani \\ Dipartimento di Fisica, Università "La Sapienza", I-00185 Roma, Italy
}

(August 15, 2018)

\begin{abstract}
Products of random transfer matrices are applied to low dimensional disordered systems to evaluate numerically extensive quantities such as entropy and overlap probability distribution. The main advantage is the possibility to avoid numerical differentiation. The method works for arbitrary disorder distributions at any temperature.
\end{abstract}

75.10.Nr, 05.50.+q, 02.50.+s

Typeset using REVTEX 
Products of random transfer matrices are a powerful tool for the study of low dimensional systems [1]. Up to now they have been mainly used to evaluate free energies and correlation functions. From a formal point of view, the other thermodynamical quantities can be obtained by differentiating the free energy with respect to suitable parameters. However, in most of the cases, one has only numerical solutions and, expecially at low temperatures, a very high precision is required for a precise differentiation. In practice, it is very difficult to estimate quantities such as the entropy or the specific heat even in a one dimensional disordered system.

The purpose of our letter is to introduce a new numerical method to evaluate thermodynamical quantities directly from the products of random transfer matrices avoiding numerical differentiation. The method is motivated by the papers of Masui et al. [2, 3] on the study of the number of metastable states in one and two dimensional Ising disordered systems. As specific example we shall discuss the overlap probability distribution $P(q)$ for zero and finite temperature for a one dimensional Ising chain. The method can be extended to higher dimensional systems with short range interactions.

An Ising chain consists of $N$ Ising spins with nearest neighbour interactions in an external field. The interactions and/or the local field are random quenched variables. At zero temperatures the only states which are important are the metastable states in which the spin $\sigma_{i}$ is aligned with the total magnetic field $h_{i}$ acting on it. If we denote by $\sigma_{i}^{\alpha}$ the spin configuration in the metastable state $\alpha$, the overlap between pairs of states is $q_{\alpha \beta}=(1 / N) \sum_{i} \sigma_{i}^{\alpha} \sigma_{i}^{\beta}$ where $\alpha$ and $\beta$ denote a pair of metastable states, and the sum is extended to all the $N$ spins. In general $q_{\alpha \beta}$ depends on the realization of disorder and on the pair of states. The number of metastable states with a given overlap can be obtained from [4, [0]

$$
\mathcal{N}_{N}(\omega, \beta)=\sum_{\sigma^{1}} \sum_{\sigma^{2}} e^{N \omega q_{12}} \prod_{i}^{1, N} \prod_{\alpha}^{1,2} \theta\left(h_{i}^{\alpha} \sigma_{i}^{\alpha}\right) e^{-\beta H^{\alpha}} .
$$

where $H^{1}$ and $H^{2}$ are the hamiltonian of two identical replicas of the system. The step $\theta$ functions ensures that only the metastable states are counted. The Gibbs weight $\exp \left(-\beta H^{\alpha}\right)$ has been introduced to select the energy of the metastable states. For any given value of $\beta$ 
the sum Eq. (1) will be dominated by metastable states of defined energy. In the same way any given value of $\omega$ selects metastable states of defined overlap. Due to the $\theta$ function in Eq. (11), the parameter $\beta$ is not necessary related to the temperature, but it can be seen as a Lagrange multiplier.

$\mathcal{N}_{N}(\omega, \beta)$, the number of metastable states of a chain of length $N$ for a given value of $\beta$ and $\omega$, can be written as a sum over the energies and overlaps

$$
\mathcal{N}_{N}(\omega, \beta)=\sum_{q, E} \mathcal{N}_{N}(q, E) e^{-2 \beta E+N \omega q} .
$$

For large $N$ the sum is dominated by the saddle point energy $E^{\prime}$ and overlap $q^{\prime}$, so that we can write

$$
\mathcal{N}_{N}(\omega, \beta) \sim \mathcal{N}_{N}\left(q^{\prime}, E^{\prime}\right) e^{-2 \beta E^{\prime}+N \omega q^{\prime}}
$$

Equation (11) can be written as a product of random matrices, so that the Oseledec theorem ensures that in the thermodynamic limit the quantity in Eq. (3) is the same for almost all realizations of disorder [6,1]. Consequently one has the Legendre transform

$$
\frac{1}{N} \overline{\ln \mathcal{N}_{N}(q, \epsilon)}=\frac{1}{N} \overline{\ln \mathcal{N}_{N}(\omega, \beta)}+2 \beta \epsilon-\omega q
$$

where the bar denotes the average over the realizations of disorder. Here $\epsilon \equiv \epsilon(\omega, \beta)$ is the energy per spin and $q=q(\omega, \beta)$ the overlap selected by the chosen value of $\omega$ and $\beta$. In particular the average overlap is obtained as [5]

$$
q(\omega=0, \beta)=\bar{q} \equiv \int q \overline{P(q)} d q
$$

and is the value of $q$ where $\lim _{N \rightarrow \infty}(1 / N) \overline{\ln N_{N}(q, \epsilon)}$ reaches its maximum value.

The key point is that not only $(1 / N) \overline{\ln \mathcal{N}_{N}(\omega, \beta)}$ but also $q(\omega, \beta)$ and $\epsilon(\omega, \beta)$ can be computed directly from a product of transfer matrices.

We shall denote by $\boldsymbol{S}_{i} \equiv\left(\sigma_{i}^{1}, \sigma_{i}^{2}\right)$ the pair of spins at the same site in two replicas of the system. Equation (四) can then be written as a chain sum over $\boldsymbol{S}_{i}$, i.e. as a product of the $\operatorname{matrix}\left\langle\boldsymbol{S}_{i+1}\left|\hat{N}_{i}\right| \boldsymbol{S}_{i}\right\rangle$. From Eq. (1) it is easy to see that $\hat{N}_{i}$ obeys the recursion relation 


$$
\begin{aligned}
\left\langle\boldsymbol{S}_{i+1}\left|\hat{N}_{i}\right| \boldsymbol{S}_{i}\right\rangle= & \sum_{\boldsymbol{S}_{i-1}}\left\langle\boldsymbol{S}_{i}\left|\hat{N}_{i-1}^{\prime}\right| \boldsymbol{S}_{i-1}\right\rangle e^{\omega \sigma_{i}^{1} \sigma_{i}^{2}} \\
& \times \prod_{\alpha}^{1,2} \theta\left(h_{i}^{\alpha} \sigma_{i}^{\alpha}\right) e^{-\beta E_{i}^{\alpha}}
\end{aligned}
$$

with an initial matrix $\hat{N}_{0}^{\prime}$ with all elements equal and with their sum equal to unity. Here $E_{i}^{\alpha}$ is the energy of the spin $\sigma_{i}^{\alpha}$. The matrix $\hat{N}_{i-1}^{\prime}$ is obtained by rescaling $\hat{N}_{i-1}$ by the factor $n_{i-1}$ given by the sum of all its elements. This ensures that the sum of the elements of $\hat{N}_{i-1}^{\prime}$ is one.

The matrix $\hat{N}_{i}$ gives the number of metastable states of a chain of $i$ spins for all the possible configurations of $\boldsymbol{S}_{i}$ and $\boldsymbol{S}_{i+1}$. Thus the logarithm of the number of metastable states per spin, averaged over the disorder, is

$$
\frac{1}{N} \overline{\ln \mathcal{N}_{N}(\omega, \beta)}=\lim _{N \rightarrow \infty} \frac{1}{N} \sum_{i=1}^{N} \ln n_{i}
$$

The value of $q$ and $\epsilon$ is obtained from the derivative of $\mathcal{N}_{N}(\omega, \beta)$ with respect to $\omega$ and $\beta$, respectively. These can be expressed in terms of the matrices derivative of $\hat{N}_{i}$. For example, defining the matrix $\hat{O}_{i}$ as $\left\langle\boldsymbol{S}_{i+1}\left|\hat{O}_{i}\right| \boldsymbol{S}_{i}\right\rangle \equiv(\partial / \partial \omega)\left\langle\boldsymbol{S}_{i+1}\left|\hat{N}_{i}\right| \boldsymbol{S}_{i}\right\rangle$ the derivative of Eq. (6) yields recursion relation

$$
\begin{aligned}
\left\langle\boldsymbol{S}_{i+1}\left|\hat{O}_{i}\right| \boldsymbol{S}_{i}\right\rangle= & \sum_{\boldsymbol{S}_{i-1}}\left[\left\langle\boldsymbol{S}_{i}\left|\hat{O}_{i-1}^{\prime}\right| \boldsymbol{S}_{i-1}\right\rangle\right. \\
& \left.+\sigma^{1} \sigma^{2}\left\langle\boldsymbol{S}_{i}\left|\hat{N}_{i-1}^{\prime}\right| \boldsymbol{S}_{i-1}\right\rangle\right] \\
& \times e^{\omega \sigma_{i}^{1} \sigma_{i}^{2}} \prod_{\alpha=1,2} \theta\left(h_{i}^{\alpha} \sigma_{i}^{\alpha}\right) e^{-\beta E_{i}^{\alpha}}
\end{aligned}
$$

with the initial condition

$$
\left\langle\boldsymbol{S}_{2}\left|\hat{O}_{1}\right| \boldsymbol{S}_{1}\right\rangle=\sigma_{1}^{1} \sigma_{1}^{2}\left\langle\boldsymbol{S}_{2}\left|\hat{N}_{1}\right| \boldsymbol{S}_{1}\right\rangle
$$

The matrix $\hat{O}_{i-1}^{\prime}$ is related to $\hat{O}_{i-1}$ as

$$
\left\langle\boldsymbol{S}_{i+1}\left|\hat{O}_{i}^{\prime}\right| \boldsymbol{S}_{i}\right\rangle=\left[\left\langle\boldsymbol{S}_{i+1}\left|\hat{O}_{i}\right| \boldsymbol{S}_{i}\right\rangle-o_{i}\left\langle\boldsymbol{S}_{i+1}\left|\hat{N}_{i}^{\prime}\right| \boldsymbol{S}_{i}\right\rangle\right] / n_{i}
$$

where $o_{i}$ is the sum of the elements of $\hat{O}_{i}$. 
From the renormalization Eq. (10) follows that the value of $q$ for the chosen value of $\omega$ and $\beta$ is given by the weighted average of $o_{i}$ as

$$
q=\lim _{N \rightarrow \infty} \frac{1}{N} \sum_{i=1}^{N} \frac{o_{i}}{n_{i}} .
$$

A similar equation yields $\epsilon$.

The method can be generalized to finite temperatures. In this case we have to count all the states, and not just the metastable ones. Thus We should thus eliminate the $\theta$-functions in the above equations. Moreover, in this case $\beta^{-1}$ is the temperature of the system.

In the absence of the $\theta$-functions, which relates the states in $i-1, i$ and $i+1$, we can use a simpler algorithm [7]. The equations are formally the same, but with matrices replaced by vectors. Therefore, in analogy with the zero temperature case, we introduce the vector $\boldsymbol{N}_{i}\left(\boldsymbol{S}_{i+1}\right)$ which now obeys the recursion relation

$$
\boldsymbol{N}_{i}\left(\boldsymbol{S}_{i+1}\right)=\sum_{\boldsymbol{S}_{i}} e^{-\beta E_{i}^{1}-\beta E_{i}^{2}+\omega \sigma_{i}^{1} \sigma_{i}^{2}} \boldsymbol{N}_{i-1}^{\prime}\left(\boldsymbol{S}_{i}\right)
$$

where the initial vector $\boldsymbol{N}_{0}^{\prime}\left(\boldsymbol{S}_{1}\right)$ has all the elements equal to one. At each iteration the vector is normalized so that the sum of the elements of $\boldsymbol{N}_{i-1}^{\prime}\left(\boldsymbol{S}_{i}\right)$ is one. To evaluate $q$ and $\epsilon$ we introduce the vectors of the derivatives of $\boldsymbol{N}_{i}\left(\boldsymbol{S}_{i+1}\right)$ with respect to $\omega$ and $\beta$, respectively. For example, taking the derivative with respect to $\omega$ of Eq. (12) leads to the vector $\boldsymbol{O}_{i}\left(\boldsymbol{S}_{i+1}\right)=(\partial / \partial \omega) \boldsymbol{N}_{i}\left(\boldsymbol{S}_{i+1}\right)$ obeying the recursion relation

$$
\begin{aligned}
\boldsymbol{O}_{i}\left(\boldsymbol{S}_{i+1}\right)= & \sum_{\boldsymbol{S}_{i}}\left[\boldsymbol{O}_{i-1}^{\prime}\left(\boldsymbol{S}_{i}\right)+\sigma_{i}^{1} \sigma_{i}^{2} \boldsymbol{N}_{i-1}^{\prime}\left(\boldsymbol{S}_{i}\right)\right] \\
& \times e^{\omega \sigma_{i}^{1} \sigma_{i}^{2}} \prod_{\alpha}^{1,2} e^{-\beta E_{i}^{\alpha}}
\end{aligned}
$$

with initial condition $\boldsymbol{O}_{0}^{\prime}\left(\boldsymbol{S}_{1}\right)=0$. The vector $\boldsymbol{O}_{i}$ and $\boldsymbol{O}_{i}^{\prime}$ are related by

$$
\boldsymbol{O}_{i}^{\prime}\left(\boldsymbol{S}_{i+1}\right)=\left[\boldsymbol{O}_{i}\left(\boldsymbol{S}_{i+1}\right)-o_{i} \boldsymbol{N}_{i}^{\prime}\left(\boldsymbol{S}_{i+1}\right)\right] / n_{i}
$$

where as above $n_{i}$ and $o_{i}$ are the sum of the elements of $\boldsymbol{N}_{i}\left(\boldsymbol{S}_{i+1}\right)$ and $\boldsymbol{O}_{i}\left(\boldsymbol{S}_{i+1}\right)$, respectively. The average energy $\epsilon$ is evaluated in a similar way. 
The specific heat and susceptibility can also be computed with this method by introducing the matrix or the vector of second derivative.

We have analyzed only Ising chains, but our arguments apply to any formally one dimensional system and can be easily generalized to two and three dimensional systems by considering strips and bars, respectively. The numerical calculation becomes, however, very heavy since the dimension of the transfer matrices and vectors grows exponentially with the transversal dimension of the system.

Let us apply this method to the Ising chain in a random field described by the hamiltonian

$$
H=-\sum_{i=1}^{N} J \sigma_{i} \sigma_{i+1}-\sum_{i=1}^{N} h_{i} \sigma_{i}
$$

where $h_{i}$ is a random local field. Without loosing in generality we can take $J=1$. It is known [8.9] that if $h_{i}$ are distributed with probability distribution

$$
\mathcal{P}\left(h_{i}\right)=p \delta\left(h_{i}-h\right)+(1-p) \delta\left(h_{i}+h\right)
$$

with $0<p<1$ then the zero temperature entropy is different from zero. This is peculiar of distribution (16). In fact, if we replace the delta functions by two sharp peaks of width $\Delta h$, then $S(T) \propto T$ for $T \ll \Delta h$ [B]. This drastic change can be clearly seen in the behaviour of $\bar{q}=q(\omega=0, \beta=1 / T)$ as a function of $T$, as shown in Fig. 1. In Fig. 2 we report the spin glass susceptibility $\chi_{\mathrm{SG}}$, defined as the derivative of $q(\omega, \beta)$ with respect to $\omega$ for $\omega=0$, as a function of temperature for two different values of $\Delta h$. The value of $\chi_{\mathrm{SG}}$ has been obtained by using the vector of the second derivative of $\hat{N}_{i}$ with respect to $\omega$. Finally Fig. 目 shows the typical form of $\lim _{N \rightarrow \infty}(1 / N) \overline{\ln N_{N}(q, \epsilon)}$. The maximum is attained for $q=\bar{q}$. In the figures $J=1$ and $h=2$. The convergence of the method is very fast. Reasonable good numbers are obtained already with $O\left(10^{4}\right)$ iterations. For $T=0$, the results are in very good agreement with the theoretical prediction of Ref. [9].

The parabolic shape of $\lim _{N \rightarrow \infty}(1 / N) \overline{\ln N_{N}(q, \epsilon)}$ means that the overlap probability distribution $P(q)$ is selfaveraging and equal to a $\delta$-function on the average value $\bar{q}$. This is a general result for one dimensional systems with short range interactions [7]. 
We conclude by noting that since for $T \rightarrow 0$ the probability distribution $P(q)$ remains a $\delta$-function [7], the limit $T \rightarrow 0$ is not singular and the results of the zero temperature method perfectly reproduce those of the $T \rightarrow 0$ limit of the finite temperature method.

In conclusion we have described a method to evaluate all thermodynamical quantities in terms of products of random transfer matrices avoiding numerical differentiation. The method can be used, in principle, also for two and three dimensional systems. The method is not restricted to spin systems, but it can be used in any other problems which involves products of transfer matrices, e.g. directed polymers. 


\section{REFERENCES}

[1] A. Crisanti, G. Paladin and A. Vulpiani, Products of Random Matrices in Statistical Physics Springer Series in Solid State Sciences, in press (1993).

[2] S. Masui, B. W. Southern and A. E. Jacobs, Phys. Rev. B 39, 6925 (1989).

[3] S. Masui, A. E. Jacobs, C Wicentowich and B. W. Southern, J. Phys. A 26, 25 (1993).

[4] S. Franz, G. Parisi and M. A. Virasoro, J. de Physique I 2, 1869 (1992).

[5] A. Crisanti, G. Paladin, M. Serva and A. Vulpiani, Phys. Rev. Lett., submited (1993).

[6] V. I. Oseledec, Trans. Moscow Math. Soc. 19, 197 (1988).

[7] A. Crisanti, G. Paladin, M. Serva and A. Vulpiani, J. de Physique I, submited (1993).

[8] M. Puma and J. F. Fernandez, Phys. Rev. B 18, 1931 (1978).

[9] B. Derrida and H. J. Hilhorst, J. Phys. A 16, 2641 (1983). 


\section{FIGURES}

FIG. 1. The average overlap $\bar{q}$ as a function of temperature $T$ for $\Delta h=0$ (a) and $\Delta h=0.3$ (b). In both cases $J=1$ and $h=2$.

FIG. 2. The spin glass susceptibility $\chi_{\mathrm{SG}}$ as a function of temperature $T$ for $\Delta h=0$ (a) and $\Delta h=0.3(\mathrm{~b})$. In both cases $J=1$ and $h=2$.

FIG. 3. $\lim _{N \rightarrow \infty}(1 / N) \overline{\ln N_{N}(q, \epsilon)}$ as a function of $q$ for $\Delta h=0, T=0$ and $\epsilon=\epsilon_{\min }$, the ground state energy. The maximum is obtained for $q=\bar{q}$. In figure $J=1$ and $h=2$. 